\title{
УСТРАНЕНИЕ ИМПУЛЬСНЫХ ПОМЕХ И ФЛУКТУАЦИОННЫХ ШУМОВ В СИГНАЛЕ ПИРОМЕТРА НА ОСНОВЕ МЕТОДА ДВУМЕРНОГО СКАНИРОВАНИЯ
}

Шлыкөв В.В., доц., к.т.н. v.shlykov@kpi.ua

г. Киев, Украина

Данилова В.А., ст. преподаватель valnaa@ukr.net

Кафедра биомедицинской инженерии Национального технического университета «Киевский политехнический институт имени Игоря Сикорского»

\begin{abstract}
Реферат - Предложен метод предварительной обработки циифровых данных, полученых при регистрации температуры биологического объекта пирометром, который позволяет устранить в полезном сигнале флуктуационные шумы и импульсные помехи, обусловленные различной регулярной структурой геметрии фотоэлектронных элементов оптической матрицы или структурой канала передачи сигнала.
\end{abstract}

Ключевые слова - температурный контроль, флуктуационный шум, импульсная помеха, подавление шумов, пирометр, кардиохирургия, биологический объект.

\section{І. ВВЕДЕНИЕ}

В современных исследованиях цифровые данные являются основным видом информации. Применение цифрового оборудования в различных условиях биомедицинских исследований может приводить к появлению различных эффектов в регистрируемом сигнале, в том числе шумов. Для эффективного устранения шума, который обусловлен различной регулярной структурой геметрии фотоэлектронных элементов оптической матрицы или структурой канала передачи сигнала в пирометрической системе, требуется знать его тип. Существуют разные типы шумов, например, гауссовский шум (цифровые изображения с тепловизионных камер), пуассоновский шум (рентгеновские снимки), спеклшум (сигналы сонно- и электроэнцефалограмм) и т.д.

В настоящее время разработано много специальных методов устранения шумов для случаев, когда известен тип шума. Например, метод полной вариации и метод медианной фильтрация одномерных сигналов [1 - 13], которые яляются известными и эффективными подходами для устранения гауссовского и пуассоновского шума $[12,13]$. Оба типа шумов (пуассоновский и гауссовский) хорошо изучены, но также важна их комбинация в регистрируемом сигнале [15], которая часто присутствует в биомедицинских сигналах и изображениях, например, в сигнале пирометра и изображениях электронной микроскопии [16, 17].

Традиционный подход к подавлению таких типов шумов связан с использованием эталонных излучателей и коррекцией мультипликативных и аддитивных коэффициентов оптической матрицы [3], что обеспечивает достаточно высокую по полю зрения однородность интенсивности излучения. Однако этот подход не лишен недостатков, которые связанны со следующим:

- необходимость использования эталонных оптических излучателей (как минимум два); 
- высокие требования к однородности оптических излучателей по полю зрения;

- необходимость использования в процессе калибровки нескольких эталонных излучателей (если динамический диапазон сигналов меняется в пространстве или во времени);

- необходимость периодического повторения процедуры калибровки (если изменяется во времени спектральный состав каналов передачи и обработки сигналов).

В связи с этим в последние годы появился ряд работ, направленных на разработку алгоритмов и практическое применение идеи двумерного сканирования и микросканирования для подавления гауссовского и пуассоновского шума в информационных системах [18 - 22]. Например, концепция полной вариации для устранения гауссовского шума была впервые применена в работе [12] на основе использования полной вариации в задачах обработки изображений (ROF-модель). Эта модель предназначена для устранения гауссовского шума $[12,13]$, однако может быть использована для устранения и других типов шумов.

Как средство предварительной обработки цифровых данных достаточно часто применяются на практике также медианные фильтры [23 - 27]. Характерной особенностью медианного фильтра является его нелинейность, которая проявляктся в избирательности фильтра по отношению к последовательности данных измерения, представляющих собой немонотонную составляющую сигнала в пределах окна (апертуры) фильтра, и резко выделяющихся на фоне соседних отсчетов. В то же время медианный фильтр не действует на монотонную составляющую сигнала, оставляя эту последовательность данных без изменений.

C учетом выше изложенного, для повышения достоверности контроля температуры сердца в условиях искусственного кровообращения с использованием пирометра необходимо разработать подход, позволяющий устранить импульсные помехи и флуктуационные шумы в канале измерения температуры. Актуальность постановки такой задачи определяется не только техническими особенностями оптической матрицы пирометра, а также программным алгоритмом подавления геометрического шума по регистрируемым сигналам, что расширяет возможнлсти применения полученных результатов в других областях биомедицинских исследованияз [28].

\section{ІІ. ЦЕЛЬ ИССЛЕДОВАНИЙ}

Целью настоящей работы является разработка комплексной математической модели и програмного алгоритма устранения в полезном сигнале пирометра флуктуационных шумов и импульсных помех, которые обусловленны различной регулярной структурой геметрии фотоэлектронных элементов оптической матрицы и структурой канала передачи сигнала.

Реализация неинвазивного контроля температуры в тканях изолированного сердца была выполнена в условиях, которые приближены к условиям искусственного кровообращения.

В экспериментах был использован реальный сигнал с пиромеира после добавления к нему шума. Качество программной обработки сравнивалось с другими методами устранения шума, например: ROF-модель, модифицированная ROF-модель и фильтрация медианой [29]. Предполагается, что такая математическая модель одномерного сигнала должна эффективно устранить комбинированный гауссовский и пуассоновский шум с учётом пропорции этих типов шумов.

\section{III. ИНСТРУМЕНТАЛЬНЫЕ СРЕДСТВА ИССЛЕДОВАНИЯ}

Исходным материалом для реализации метода устранения шумов на основе двумерного сканирования является оптическая матрица фотоэлектронных елементов пирометра Opris MS Plus (Optris $\mathrm{GmbH}$, Германия) с числом элементов в матрице $N=2^{M}$, где $\mathrm{M}$ - число строк (столбцов) пьезоэлектрической матрицы. Используемая пирометрическая система безконтактного контроля температуры на основе пирометра Opris MS Plus обеспечивает 
измерение температуры на расстоянии до объекта не более $140 \mathrm{~mm}$ в диапазоне температур от $-5^{\circ} \mathrm{C}$ до $40^{\circ} \mathrm{C}$ в спектральном диапазоне 8-14мкм с погрешностью не более $\pm 0,5 \%$ или $\pm 0,7^{\circ} \mathrm{C}$ от диапазона температур.

\begin{tabular}{lccr}
\multicolumn{1}{c}{ С целью } & упрощения & алгоритма \\
вычислений & и & выявления & основных \\
особенностей & изучаемого & подхода \\
использовалась & следующая & система
\end{tabular}
приближений:

1. Оптическая матрица пирометра имеет различную форму и геометрические размеры, однако, с точки зрения простоты реализации модели, выбираем квадратную форму, с одинаковым размером и ориентацией пьезоэлементов;

2. Кроме полезного сигнала регистрируемые данные содержат флуктуационные шумы и импульсные потоки различной интенсивности с регулярной или хаотической структурой (гауссовский и пуассоновский шум);

3. Под импульсным шумом понимается искажение регистрируемых сигналов большими импульсными выбросами произвольной полярности и малой длительности, причиной которых являются как внешние импульсные электромагнитные помехи, так и наводки, сбои и помехи в работе системы.

В качестве подопытного материала использовано изолированное сердце весом до 500 г, которое находилось в термозберегающей емкости. В серце была выделена безымянная артерия, по которой в восходящую аорту введена полиэтиленовая канюля артериальной магистрали препарата для региональной перфузии сердца. В начале эксперимента сердце на некоторое время было погружено в теплую дистиллированную воду, чтобы его температура достигла примерно $25^{\circ} \mathrm{C}$. Затем в отделенную восходящую аорту ствола легочной артерии выполнена коронарная перфузия. Врезультате, постепенно температура сердца снижалась до $17^{\circ} \mathrm{C}$, что соответствует его температуре в условиях искусственного кровообращения.

Разработанный на этой основе программный алгоритм подавления импульсных помех, флуктационного и геометрического шума в регистрируемом сигнале температуры обладает свойством универсальности в условиях искусственного кровообращения [30].

\section{IV. МОДЕЛЬ УСТРАНЕНИЯ СМЕСИ ШУМОВ НА ОСНОВЕ МЕДИАННОЙ ФИЛЬТРАЦИИ СИГНАЛОВ}

Применение медианного фильтра для устранения смеси шумов является более эффективным по сравнению с линейными фильтрами, поскольку процедуры линейной фильтрации являются оптимальными при равномерном или гауссовом распределении помех, что предполагает упрощение особенностей структуры шумов в реальных сигналах. Эффективным применение медианного фильтра оказывается при очистке регистрируемых сигналов от импульсных шумов при обработке изображений, акустических сигналов, передаче сигналов от тепловых приемников излучения и т.п.

Медианная фильтрация реализуется в виде алгоритма локальной обработки временных отсчетов в скользящем окне, которое включает определенное число отсчетов сигнала. Для каждого положения скользящего окна, выделенные в нем отсчеты, ранжируются по возрастанию или убыванию значений. Медианой в ранжированном списке является группа отсчетов, которая по своему положению находиться в середине списка.

C учетом ранжирования отсчетов в списке медианные фильтры хорошо подавляют шумы и помехи, протяженность которых составляет менее половины скользящего окна. Полезным сигналом является сиабильная последовательность данных (в случае одномерного сигнала) или массив данных (в случае двумерного сканирования), которая не изменяется при медианной фильтрации.

При регистрации температуры пирометром и обработке данных предполагается, что измеренный сигнал $\mathrm{x}(\mathrm{t})$ кроме полезного сигнала $\mathrm{s}\left(\mathrm{t}-\tau_{0}\right)$ и флуктуационных шумов $\mathrm{q}(\mathrm{t})$ содержит также импульсные потоки $\mathrm{g}(\mathrm{t})$ различной интенсивности с регулярной или хаотической структурой:

$$
x(t)=s\left(t-\tau_{0}\right)+g(t)+q(t) .
$$


где $\mathrm{s}\left(\mathrm{t}-\tau_{0}\right)$ - полезный сигнал, который имеет неизвестное временное положение $\tau_{0} \in$ $[0, \mathrm{~T}]$,

$\mathrm{q}(\mathrm{t})$ - флуктуационные шумы $\mathrm{c}$ регулярной или хаотической структурой (гауссовский и пуассоновский шум),

$$
\mathrm{g}(\mathrm{t})=\sum_{\mathrm{k}} \delta\left(\mathrm{t}-\tau_{\mathrm{k}}\right)-\text { импульсные потоки }
$$

различной интенсивности с регулярной или хаотической структурой, $\tau_{\mathrm{k}}-$ неизвестное временное положение импульсов,

Согласно теории вероятностей, функция распределения $\mathrm{F}(\mathrm{x})$ измеренных величин $\mathrm{x}=\operatorname{med}\left(\mathrm{x}_{1}, \ldots, \mathrm{x}_{\mathrm{n}}\right)$ для больших $\mathrm{n}$ является приблизительно нормальной:

$$
\mathrm{F}(\mathrm{x})=\mathrm{N}\left(\mathrm{m}_{\mathrm{t}}, \sigma_{\mathrm{n}}\right),
$$

где $\mathrm{m}_{\mathrm{t}}-$ теоретическая медиана, определяемая из условия $\mathrm{F}\left(\mathrm{m}_{\mathrm{t}}\right)=0.5, \sigma_{\mathrm{n}}-$ дисперсия распределения, $\mathrm{n}$ - число измерений величины х.

При этом дисперсия распределения медианы определяктся:

$$
\sigma_{\mathrm{n}}^{2}=1 /\left(\mathrm{n} 4 \mathrm{f}^{2}\left(\mathrm{~m}_{\mathrm{t}}\right)\right)
$$

где $\mathrm{f}(\mathrm{x})$ - распределение плотности вероятностей величин х.

Если случайные величины х являются независимыми и одинаково распределенными с нормальным распределением $\mathrm{N}(\mathrm{m}, \sigma)$, то $\mathrm{m}_{\mathrm{t}}$ $=\mathrm{m}$, и формула дисперсии медианы для малых нечетных значений $\mathrm{n}$ принимает вид:

$$
\sigma_{\mathrm{g}}^{2} \approx \pi \sigma^{2} /(2 \mathrm{n}-2+\pi)
$$

Если функция $\mathrm{f}(\mathrm{x})$ симметрична относительно $\mathrm{m}$, то распределение медиан будет также симметрично относительно $\mathrm{m}_{\mathrm{t}}$. Таким образом, будет справедлива следующая формула для определения математического ожидания:

$$
M\left\{\operatorname{med}\left(x_{1}, \ldots, x_{n}\right)\right\}=M\left\{x_{i}\right\}=m .
$$

Следует также учитывать, что при медианной фильтрации сигнала степень сглаживания финкции зависит от размеров апертуры фильтра и формы окна.

Критерий, в соответсвии с которым производится

увеличение

размера

скользящего окна с центральным отсчетом $\mathrm{x}_{\mathrm{r}}$ и выполняется следующая итерация, имеет вид:

$$
\max \left[\mathrm{S}_{\mathrm{n}}\left(\mathrm{x}_{\mathrm{r}}\right)\right]<\mathrm{S}_{\text {порог }} .
$$

где $\mathrm{S}_{\mathrm{n}}\left(\mathrm{x}_{\mathrm{r}}\right)-$ величина отклонения амплитуды соседних отсчетов сигнала $\mathrm{x}(\mathrm{t})$, попавших в скользящее окно размером $\mathrm{n}_{\mathrm{t}}$, относительно амплитуды центрального отсчета x (r),

$\mathrm{S}_{\text {порог }}=\left[\begin{array}{ll}0, & \mathrm{~T}\end{array}\right]-$ пороговый коэффициент интенсивности импульсного потока.

На рис. 1 представлен пример применения медианной фильтрации к модельному сигналу $\mathrm{a}_{\mathrm{k}}$, который составлен из детерминированного сигнала $\mathrm{s}_{\mathrm{k}}$ и случайного сигнала $\mathrm{q}_{\mathrm{k}}$, имеющего нормальное распределение с одиночными импульсными выбросами $\delta(t)$ малой длительности. Окно медианного фильтра составляет $\mathrm{n}_{\mathrm{t}}=5$ отсчетов. Результат медианной фильтрации представлен в виде отсчетов $b_{k}$.

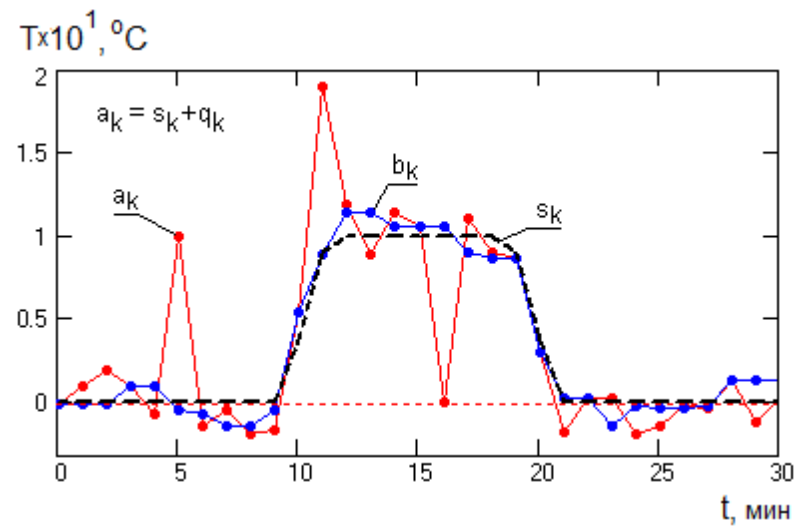

Рис. 1. Пример применения медианной фильтрации к модельному сигналу: $\mathrm{a}_{\mathrm{k}}$ - модельный сигнал, $b_{k}-$ результат медианной фильтрации.

В качестве начальных и конечных условий медианной фильтрации приняты конечные значения модельного сигнала $\mathrm{a}_{\mathrm{k}}$, или медиана $\mathrm{m}_{\mathrm{t}}$, которая вычисляется для точек, вписаных в пределы апертуры.

Приведенные результаты вычисления медианы $\mathrm{m}_{\mathrm{t}}$ для сигнала $\mathrm{x}(\mathrm{t})$ справедливы как для одномерной, так и для двумерной фильтрации, если число отсчетов n выбирать равным числу точек в апертуре медианного фильтра. 


\section{V. МОДЕЛЬ УСТРАНЕНИЯ СМЕСИ ШУМОВ НА ОСНОВЕ МЕТОДА ПОЛНОЙ ВАРИАЦИИ}

Случайный процесс, который может представляется белым шумом, имеет спектральную плотность мощности $S_{\eta}(\omega)$ постоянную во всей частотной области, а его средняя мощность (дисперсия) бесконечна. Поэтому белый шум $\xi(t)$ не является физически реальным процессом, но представляет собой удобную математическую абстракцию.

Модель нормального случайного процесса на основе метода полной вариации основывается на двух положениях теории случайных процессов:

1. Результатом произвольного линейного преобразования гауссовского случайного процесса является случайный гауссовский процесс;

2. Спектральные плотности мощности случайных процессов на входе $S_{в x}(\omega)$ и выходе $S_{\text {вых }}(\omega)$ линейного фильтра с частотной характеристикой $H(i \omega)$ связаны соотношением:

$$
\begin{aligned}
& S_{\text {выx }}(\omega)=|H(i \omega)|^{2} S_{\text {вx }}(\omega), \\
& H(i \omega)=\left\{\begin{array}{l}
1,\left|\omega-\omega^{\prime}\right| \leq \Delta \omega / 2 \\
0,\left|\omega-\omega^{\prime}\right|>\Delta \omega / 2
\end{array},\right.
\end{aligned}
$$

где $\omega^{\prime}, \Delta \omega-$ средняя частота и ширина полосы пропускания фильтра в полосе частот $\left[\omega^{\prime}-\Delta \omega / 2, \omega^{\prime}+\Delta \omega / 2\right]$.

Для решения задачи моделирования методом вариации необходимо, чтобы спектральная плотность мощности для случайного процесса $S_{\eta}(\omega)$ описывалась дробно-рациональной функцией от переменной $\omega$.

Если на вход линейного фильтра поступает белый шум $\xi(t)$, то спектральная плотность мощности случайного ипроцесса на входе будет равна:

$$
S_{\eta}(\omega)=\frac{N_{0}}{2},|\omega|<\infty,
$$

Тогда, функцию спектральной плотности мощности на выходе линейного фильтра можно представить в дробнорациональном виде:

$$
\begin{gathered}
S_{\xi}(\omega)=\frac{b_{M} \omega^{2 M}+b_{M-1} \omega^{2(M-1)}+\cdots+b_{0}}{a_{N} \omega^{2 N}+a_{N-1} \omega^{2(N-1)}+\cdots+a_{0}},(10) \\
\text { где } \alpha_{n} \text { и } \beta_{m}-\text { корни полиномов, }
\end{gathered}
$$

стоящие в числителе и знаменателе функции спектральной плотности мощности белого шума $S_{\eta}(\omega)$.

В основе алгоритма моделирования гауссовского случайного процесса с заданными спектральными свойствами находиться пропускание модельного белого шума $\xi(t)$ с единичной спектральной плотностью $S_{\eta}(\omega)=1 \quad$ через линейный фильтр, частотная характеристика которого соответствует выражению (рис. 2):

$$
\begin{aligned}
& H(s)=\frac{F(s)}{G(s)}, \text { где } \\
& F(s)=\sqrt{\frac{b_{M}}{a_{N}}} \prod_{m=1}^{M}\left(s+f_{m}\right), \\
& G(s)=\prod_{n=1}^{N}\left(s+g_{n}\right), \\
& g_{n}=i \alpha_{n}, n=\overline{1, N} \\
& f_{m}=i \beta_{m}, m=\overline{1, M}
\end{aligned}
$$

где $a_{N}$ и $b_{M}-$ положительные числа в функции спектральной плотности мощности $H(s)$ случайного стационарного процесса, , $s$ - комплексная переменная, которая соответсвует действительной частоте сигнала $\omega=i s$.
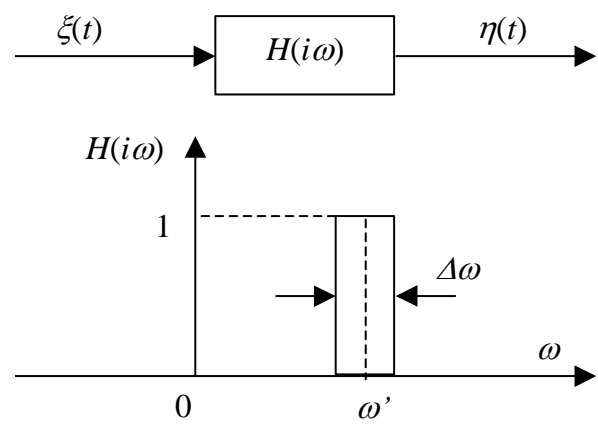

Рис. 2. Функции спектральной плотности мощности $H(s)$ линейного фильтра 
Если на вход такого линейного фильтра подать белый шум $\xi(t)$ с единичной спектральной плотностью $N_{0} / 2=1$, то на выходе фильтра сигнал будет иметь нормальное распределение. Тогда частотная характеристика филтра $H(i \omega)=|H(i \omega)| \quad$ и импульсная характеристика линейного фильтра принимает вид:

$$
h(t)=\frac{1}{2 \pi} \int_{-\infty}^{+\infty}|H(i \omega)| e^{i \omega t} d \omega,
$$

При создании цифровой модели сигнала с шумом $\xi(t)$ необходимо учитывать два основных свойства белого шума: постоянство спектральной плотности мощности $S_{\eta}(\omega)$ в частотной области и статистическую независимость временных отсчетов, которые берутся в произвольные моменты времени $\Delta t$.

Известно, что при дискретизации непрерывного сигнала $x(t)$ с периодом отсчетов $\Delta t$, спектральная функция дискретизированного сигнала становиться периодической с периодом $1 / \Delta t$. Поэтому, если спектральная плотность мощности $S_{\eta}(\omega)$ дискретного белого шума $\xi(t)$ будет постоянна на интервале $\left[\begin{array}{ll}-0.5 / \Delta t & -0.5 / \Delta t\end{array}\right]$ и равна $N_{0} / 2$, то она будет постоянна и во всей частотной области. Тогда, дисперсия случайного процесса с равномерной функцией спектральная плотности мощности $S_{\eta}(\omega)$ будет определяться по формуле:

$$
\sigma^{2}=\frac{1}{2 \pi} \int_{-0,5 / \Delta t}^{0,5 / \Delta t} \frac{N_{0}}{2} d \omega=\frac{1}{2 \pi \Delta t} \frac{N_{0}}{2} .
$$

Для единичной спектральной плотности $S_{\eta}(\omega)=N_{0} / 2=1$ получаем, что дискретная реализация белого шума $\xi(n)$ для $N$ отсчетов должна быть выполнена в соответствии с выражением:

$$
\xi(n)=\frac{1}{\sqrt{2 \pi \Delta t}} x[n], n=\overline{1, N},
$$

где $x(n)$ - случайные независимые числа с нормальным стандартным распределением (распределение Гаусса с нулевым математическим ожиданием и единичной дисперсией).

При цифровом моделировании линейного фильтра для устранения смеси щумов на основе метода вариации его входной $x(t)$ и выходной $y(t)$ сигналы представляются в виде решетчатых функций $x[n]$ и $y[n]$, которые отличны от нуля для дискретных моментов времени:

$$
t_{n}=n \Delta t, n=1, \quad 2, \quad \ldots, \quad N,(19)
$$

где $\Delta t-$ период дискретизации сигнала $x(t)$.

Выполнение точного равенства входных $x[n]=x(n \Delta t)$ и выходных сигналов $y[n]=y(n \Delta t) \quad$ для цифровой модели линейного фильтра не достижимо, на практике достигается приближенное равенство $\quad y[n] \approx y(n \Delta t) \quad$ с погрешностью, которая имеет нормальное рампределение $S_{\text {выx }}(\omega)$ с дисперсией равной:

$$
\sigma=\sqrt{1 /(2 \pi \Delta t)}
$$

Применение данного метода полной вариации к МРТ изображению сердца позволяет устранить импульсные помехи, геометрические и флуктационные шумы системы (рис. 3).

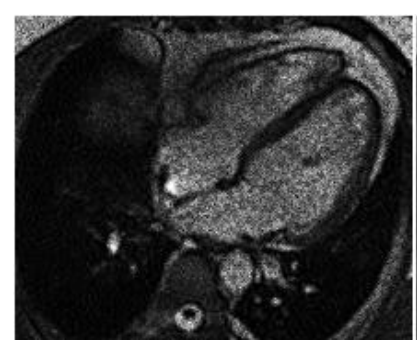

a

Рис. 3. МРТ сердца: а - изображение с белым шумом, б - очищенное изображение.

Таким образом, реализацию цифровой модели линейного фильтра для устранения смеси щумов, которые представляют случайный процесс можно реализовать на 
основе дискретного преобразования Фурье, путем выбора количество гармоник $M \approx 2 \pi / \Delta \omega$ и частоты дискретизации $\Delta t \geq M$ для случайного процесса $\xi(n), n=\overline{1, N}$.

\section{VI. МОДЕЛЬ УСТРАНЕНИЯ СМЕСИ ШУМОВ НА ОСНОВЕ МЕТОДА ДВУМЕРНОГО СКАНИРОВАНИЯ}

Величина погрешности измерения, которую дает приемник теплового излучения при регистрации температуры, существенно влияет на точность получения данных о тепловой картине на поверхности измеряемого объекта, по сравнению с эталонными измерителями (цифровой термометр, пирометр).

Новый подход к устранению смеси шумов в сигнале - импульсных помех, флуктационного и геометрического шума заключается во введении двумерного сканирования со специально подобранным законом, в соответствии с которым фотоприемные элементы (ФПЭ) матрицы смещается относительно теплового изображения. При смещении матрицы ФПЭ выполняется регистрация соответствующей последовательности кадров и их цифровая обработка. Идея модуляции параметров измерительной системы (или сигнала) с целью идентификации ее параметров уже реализована в работах исследователей, но, как показано в [31] и подтверждено в других многочисленных экспериментах [32, 33], именно двумерное сканирование позволяет решить проблему безэталонного подавления смеси шумов по сигналам фона.

Исходным материалом является квадратная матрица ФПЭ с числом элементов в строке (столбце) $N=2^{M}$ и в линейном приближении оцифрованный сигнал $A_{i, j}(\mathrm{i}, \mathrm{j})$ го от ФПЭ:

$$
A_{i j}=g_{i j} I_{i j}+h_{i j}+e_{i j}(t),
$$

где $I_{i j}-$ интенсивность излучения, падающего на ФПЭ приемника теплового излучения; $\quad h_{i j}, \quad g_{i j} \quad-$ коэффициенты преобразования, которые в данном исследовании приняты $1 ; e_{i j}(t)-$ случайная составляющая сигнала в виде гауссовского и пуассоновского шума.

Двумерное сканирование источника тепла выполняется дискретно: на пиксель вниз-вверх и на пиксель вправо-влево относительно исходного положения с достаточно малыми промежутками времени.

Полученные кадры представляются в виде системы уранений:

$$
\begin{aligned}
& \left(F_{0,0}\right)_{i j} \equiv A_{i j}=I_{i j}+h_{i j}+e_{i j}\left(t_{1}\right), \\
& \left(F_{1,0}\right)_{i j} \equiv B_{i j}=I_{i+1, j}+h_{i j}+e_{i j}\left(t_{2}\right), 1 \leq i, j \leq N(22) \\
& \left(F_{0,1}\right)_{i j} \equiv C_{i j}=I_{i, j+1}+h_{i j}+e_{i j}\left(t_{4}\right), \\
& \left(F_{0,0}\right)_{i j} \equiv A_{i j}=I_{i j}+h_{i j}+e_{i j}\left(t_{3}\right), \\
& \text { где } e_{i j}\left(t_{1}\right), \quad e_{i j}\left(t_{2}\right), \quad e_{i j}\left(t_{3}\right), \quad e_{i j}\left(t_{4}\right)-
\end{aligned}
$$

случайные величины, которые имеют нулевые среднее значение и математическое ожидание.

Для каждого пикселя теплового изображения (сигнала), который дискретно сканируется, учитывается, что случайные величины $e_{i j}\left(t_{1}\right), \quad e_{i j}\left(t_{2}\right), \quad e_{i j}\left(t_{3}\right), \quad e_{i j}\left(t_{4}\right) \quad$ в течение всего цикла сканирования $\Delta t$ остаются неизменными, не коррелируют между собой и имеют нулевые средние значения и математическое ожидание в матрице интенсивностей $\left\lfloor I_{i j}\right\rfloor$ и матрице коэффициентов $\left\lfloor h_{i j}\right\rfloor$.

После преобразования исходной системы уравнений (22) относительно коэффициентов преобразования $h_{i j}$ и $g_{i j}$, имеем:

$$
\begin{aligned}
& \left(F_{0,0}\right)_{i j}=g_{i j} I_{i j}+h_{i j}+e_{i j}\left(t_{1}\right), \\
& \left(F_{1,0}\right)_{i j}=g_{i j} I_{i+1 j}+h_{i j}+e_{i j}\left(t_{2}\right), 1 \leq i, j \leq N(23) \\
& \left(F_{0,1}\right)_{i j}=g_{i j} I_{i j+1}+h_{i j}+e_{i j}\left(t_{3}\right), \\
& \left(F_{0,0}\right)_{i j}=g_{i j} I_{i j}+h_{i j}+e_{i j}\left(t_{4}\right),
\end{aligned}
$$

Дополнив полученнкю систему уравнений условием нормирования $h_{i j}=0$ для фиксированного относительно теплового источника (i, j)-го ФПЭ, получим следующкю систему: 


$$
\left\{\begin{array}{l}
\left(F_{0,0}\right)_{i j}=g_{i j} I_{i j}+e_{i j}\left(t_{1}\right), \\
\left(F_{1,0}\right)_{i j}=g_{i j} I_{i+1 j}+e_{i j}\left(t_{2}\right), \\
\left(F_{0,1}\right)_{i j}=g_{i j} I_{i j+1}+e_{i j}\left(t_{3}\right), \\
\left(F_{0,0}\right)_{i j}=g_{i j} I_{i j}+e_{i j}\left(t_{4}\right),
\end{array}\right.
$$

В сформированной системе число уравнений $\left(4 N^{2}\right)$ существенно превышает число неизвестных $(2 N(N+1))$, при этом правые части уравнений содержат погрешности. Исключив из системы (24) все значения $\left[I_{i j}\right\rfloor, \quad$ получаем новую переопределенную систему уравнений $(2 N(N-1))$, которая позволяет определить $N^{2}$ неизвестных $\left\lfloor h_{i j}\right\rfloor$ коэффициентов:

$$
\left\{\begin{array}{c}
I_{i j}+h_{i j}=A_{i j}, \\
I_{i+1, j}+h_{i j}=B_{i j}, 1 \leq i, j \leq N, \\
I_{i j}+h_{i j}=A_{i j}, \\
I_{i, j+1}+h_{i j}=C_{i j},
\end{array}\right.
$$

Решение системы (25) относительно коэффициентов преобразования $\left\lfloor h_{i j}\right\rfloor$ позволяет скомпенсировать случайную составляющую сигнала (шум). Введенный модельный шум $e_{i j}(t)$, дает возможность достоверно оценить вкличину погрешности приемника теплового излучения при бесконтактном измерении температуры на поверхности объекта.

Применение данного метода двумерного сканирования к тепловому изображению позволяет устранить импульсные помехи, геометрические и флуктационные шумы системы (рис. 4 и 5).

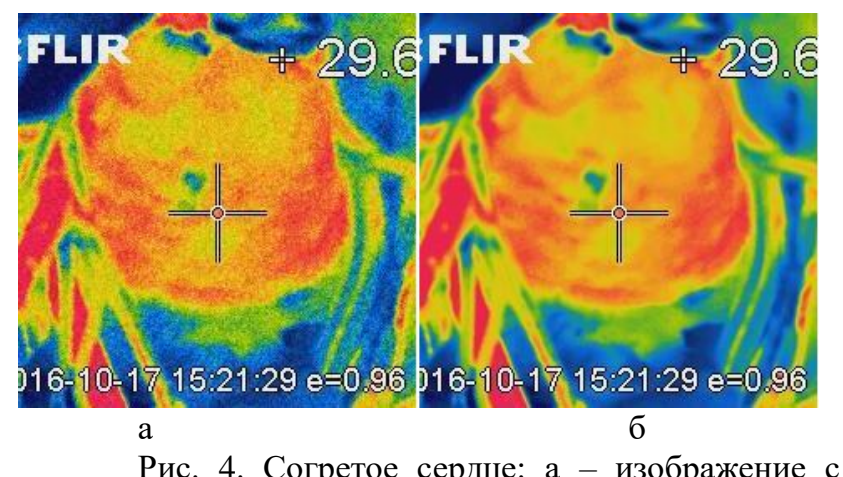
белым шумом, б - очищенное изображение.

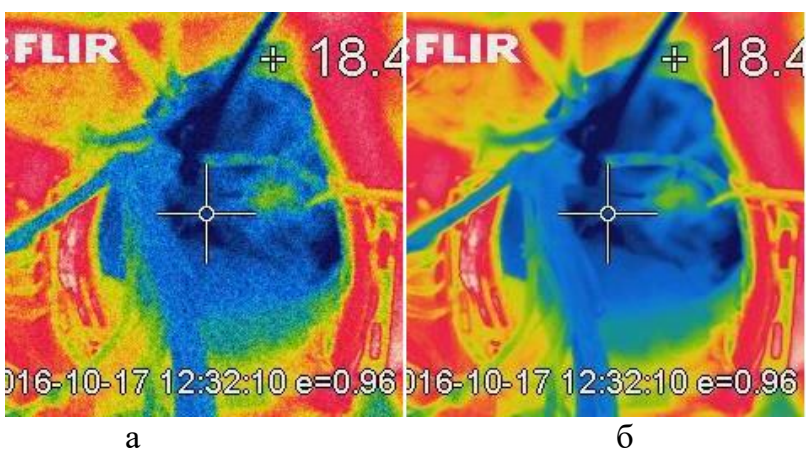

Рис. 5. Охлажденное сердце: а - изображение с белым шумом, б - очищенное изображение.

Таким образо метод устранения смеси шумов на основе двумерного сканирования позволяет подавить комплексный белый шум, который обусловлен внешними импульсными электромагнитными помехами и наводками, сбоями и помехами в работе канала передачи данных.

\section{VII. ЭКПЕРИМЕНТАЛЬНАЯ АПРОБАЦИЯ МЕТОДА ДВУМЕРНОГО СКАНИРОВАНИЯ}

В поставленном эксперименте с использованием пирометра Opris MS Plus регистрировалась температура на поверхности изолированного сердца в процессе его охлаждения и постепенного согревания, который относительно выбранного температурного режима соответствует условиям искусственного кровообращения.

На первом этапе сердце было на половину погружено в теплую воду, чтобы его температура достигла примерно $25^{\circ} \mathrm{C}$. Затем, была выделена безымянная артерия, по которой в восходящую аорту введена полиэтиленовая канюля артериальной магистрали для региональной перфузии сердца (рис. 6).

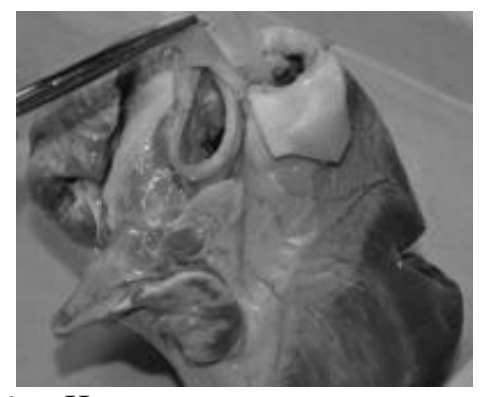

Рис. 6. Изоливанное сердце на этапе региональной перфузии 
На втором этапе экперимента была отделена восходящая аорта от общего ствола легочной артерии. Над канюлей, которая введенна в аорту была затянута тесьма турникета и начата коронарную перфузия.

На третьем, последнем этапе, после подключения капельницы к выходам канюлей была начата последовательная перфузия с помощью физиологического раствора, который предварительно был охлажден до $5^{\circ} \mathrm{C}$. Постепенно температура сердца снизилась до $17^{\circ} \mathrm{C}$, что соответствует его температуре в условиях искусственного кровообращения. Для достижения такого значения температуры охлаждения сердца были использованы два флакона с физраствором из капельниц, по 500 мл каждый.

Для измерения температуры на поверхности сердца были произвольно выбраны 4 точки. Температурные измерения фиксировались с использованием трех приборов: ртутного термометра, цифрового термометра, тепловизора.

Измерения по четырем точкам фиксировались в течение времени с 14:57 до 15:34 с интервалом 1,5 с., составив всего последовательно 16 временных отсчетов. Графики температур для охлаждения сердца во времени для температурных данных, которые получены и использованием пирометра и темловизора в точке визирования, представлены на рис. 7.

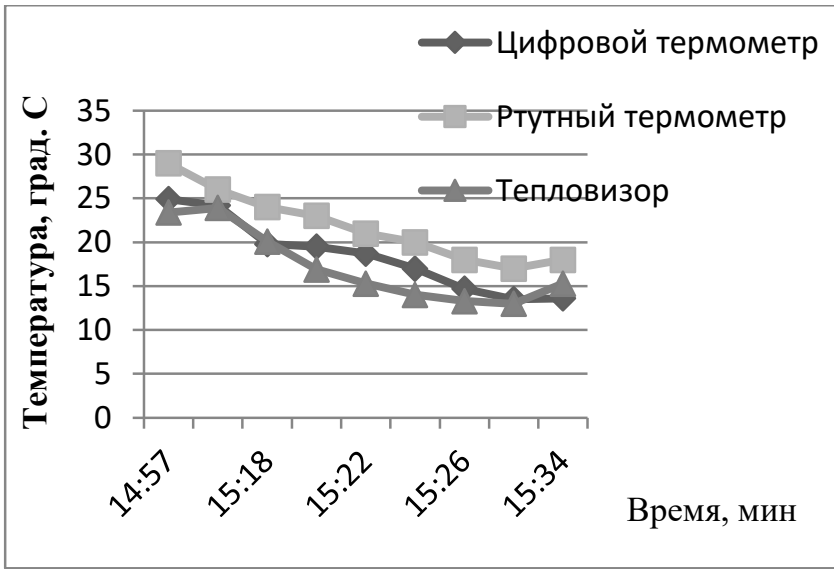
охлаждения сердца

Данные температуры из выполненого эксперимента, которые измерены в четырех точках с применением пирометра и тепловизора, и далее обработаные с применением фильтра шумов на основе метода двумерного сканирования (МДС), приведенны в таблице 1.

Таблица 1

Температурные данные *

\begin{tabular}{|c|l|l|l|l|}
\hline \multirow{2}{*}{ Время } & \multicolumn{2}{|c|}{ Пирометр, ${ }^{\circ} \mathrm{C}$} & \multicolumn{2}{c|}{ Тепловизор, ${ }^{\circ} \mathrm{C}$} \\
\cline { 2 - 5 } $14: 57$ & $T \pm \Delta T,{ }^{\circ} \mathrm{C}$ & МДС & $T \pm \Delta T,{ }^{\circ} \mathrm{C}$ & МДС \\
\hline & $23 \pm 0,5$ & 23,5 & $23 \pm 0,4$ & 23,4 \\
& $25 \pm 0,7$ & 25,8 & $23 \pm 0,8$ & 23,7 \\
& $25 \pm 0,9$ & 25,8 & $23 \pm 0,9$ & 23,9 \\
& $24 \pm 0,3$ & 24,3 & $23 \pm 0,8$ & 23,6 \\
\hline $15: 12$ & $23 \pm 0,9$ & 23,9 & $22 \pm 0,6$ & 22,6 \\
& $24 \pm 0,3$ & 24,3 & $23 \pm 0,5$ & 23,4 \\
& $24 \pm 0,2$ & 24,1 & $24 \pm 0,4$ & 24,1 \\
& $24 \pm 0,5$ & 24,5 & $23 \pm 0,9$ & 23,8 \\
\hline $15: 22$ & $13 \pm 0,2$ & 13,1 & $14 \pm 0,2$ & 14,1 \\
& $20 \pm 0,5$ & 20,5 & $18 \pm 0,5$ & 18,5 \\
& $20 \pm 0,4$ & 20,4 & $19 \pm 0,6$ & 19,6 \\
& $20 \pm 0,8$ & 20,6 & $20 \pm 0,2$ & 20,1 \\
\hline $15: 34$ & $12 \pm 0,9$ & 12,8 & $13 \pm 0,2$ & 13,1 \\
& $13 \pm 0,8$ & 13,8 & $13 \pm 0,6$ & 13.5 \\
& $13 \pm 0,9$ & 13,9 & $14 \pm 0,3$ & 14,1 \\
& $13 \pm 0,9$ & 13,8 & $15 \pm 0,2$ & 15.1 \\
\hline
\end{tabular}

В результате исследования и применения к регистрируемым данным температуры метода двумерного сканирования для устранения импульсных помех, флуктационных и геометрических шумов, удалось обнаружить и устранить случайную составляющую сигнала (шум), вызванную флуктуациями приемника теплового излкчения.

\section{VIII. ОБСУЖДЕНИЕ РЕЗУЛЬТАТОВ}

В работе проанализированы проблемы искажений в тепловых и других изображениях. Рассмотрена проблема зашумления полезного сигнала при работе матричными фотоприемными устройствами.

Описан подход к подавлению аппаратных шумов. Основными видами шума, которые удается устранить реализованными методами фильтрации, являются тепловой шум, вызванный флуктуациями температуры приемника и шум схемы оптического считывания. 
Реализована математическая модель метода двумерного сканирования для подавления импульсных помех, флуктационных и геометрических шумов, которая состоит во введении процедуры малоамплитудного сканирования со специально подобранным законом, по которому матрица ФПЭ смещается относительно изображения при регистрации последовательности кадров.

Таким образом, применение метода двумерного сканирования для устранения шумов при обработке данных, которые получены в процессе контроля температуры сердца с помощью тепловизора или цифрового пирометра позволяет повысить достоверность мониторинга температуры в миокарде в условиях искусственного кровообращения.

\section{ПЕРЕЧЕНЬ ССЫЛОК}

[1] Chan TF, Shen J. Image processing and analysis: Variational, PDE, Wavelet, and stochastic methods. SIAM, 2005; 400 p.

[2] Burger M. Level set and PDE based reconstruction methods in imaging, Springer, 2008; $319 \mathrm{p}$.

[3] Chambolle A. An introduction to total variation for image analysis. Theoretical foundations and numerical methods for sparse recovery 2009; 9: 263-340.

[4] Xu J, Feng X, Hao Y. A coupled variational model for image denoising using a duality strategy and split Bregman. Multidimensional systems and signal processing 2014; 25: 83-94. [5] Rankovic N, Tuba M. Improved adaptive median filter for denoising ultrasound images. Advances in computer science, WSEAS ECC'12 2012; 169-74.

[6] Lysaker M, Tai X. Iterative image restoration combining total variation minimization and a second-order functional. International journal of computer vision 2006; 66: 5-18.

model for image denoising and segmentation. Journal mathematical imaging and vision 2006; 26(1-2): 115-25.

Modified ROF model. Optics express 2012; 20(16): 1798718004.

[9] Tran MP, Peteri R, Bergounioux M. Denoising 3D medical images using a second order variational model and wavelet shrinkage. Image analysis and recognition 2012; 7325: 138-45.

[10] Getreuer P. Rudin-Osher-Fatemi total variation denoising using split Bregman. IPOL 2012. Source: http://www.ipol.im/pub/art/2012/g-tvd/

[11] Caselles V, Chambolle A, Novaga M. Handbook of mathematical methods in imaging, Springer, 2011. 1607 p.

[12] Rudin LI, Osher S, Fatemi E. Nonlinear total variation based noise removal algorithms. Physica D. 1992; 60: 259-68.

[13] Chen K. Introduction to variational image processing models and application. International journal of computer mathematics 2013; 90(1): 1-8.

[14] Le T, Chartrand R, Asaki TJ. A variational approach to reconstructing images corrupted by Poisson noise. Journal of mathematical imaging and vision 2007; 27(3): 257-63.

[15] Luisier F, Blu T, Unser M. Image denoising in mixed Poisson-Gaussian noise. IEEE transaction on Image processing 2011; 20(3): 696-708.

[16] Jezierska A. An EM approach for Poisson-Gaussian noise modelling. EUSIPCO 19th 2011; 62(1): 13-30.
[17] Jezierska A. Poisson-Gaussian noise parameter estimation in fluorescence microscopy imaging. IEEE International Symposium on Biomedical Imaging 9th 2012; 1663-6.

[18] Математическое моделирование коррекции неоднородности сканирующих многоэлементных фотоприемных устройств по сигналам сцены / В.Г. Морозова, С.И. Жегалов, В.Н. Соляков [и др.] // 19-я междунар. конф. по фотоэлектронике и приборам ночного видения. - М., 2006. - С. 140 - 141.

[19] Лебедев Д.Г. Моделирование адаптивного выравнивания параметров линейки фотоприемника с использованием микросканирования / Д.Г. Лебедев, К.Т. Лыонг // Информационные процессы. - 2007. - Т. 7, № 2. - С. 124 137.

[20] Соляков В.Н. Коррекция по сигналам сцены неоднородности многоэлементных фотоприемных устройств с микросканированием / В.Н. Соляков, В.Г. Морозова, С.И. Жегалов // 20-я междунар. конф. по фотоэлектронике и приборам ночного видения. - М., 2008. - С. 197 - 198.

[21] Метод коррекции неоднородности многоэлементных фотоприемных устройств по сигналам сцены / В.Н. Соляков, С.И. Жегалов, Л.Д. Сагинов [и др.] // Прикладная физика. 2008. - № 1. - С. 60 - 71 .

[21] Соляков В.Н. Метод коррекции неоднородности по сигналам сцены многоэлементных фотоприемных устройств с микросканированием / В.Н. Соляков, С.И. Жегалов, В.Г. Морозова // Прикладная физика. - 2009. - № 5. - С. 99 - 106.

[22] Соляков В.Н. Результаты практического моделирования коррекции неоднородности многоэлементных фотоприемных устройств по сигналам сцены / В.Н. Соляков, С.И. Жегалов, В.Г. Морозова // Прикладная физика. - 2009. - № 5. - С. 107 -112 .

[23] Большаков И.А., Ракошиц В.С. Прикладная теория случайных потоков, М.: Сов. радио, 1978,- $248 \mathrm{c}$.

[24] Хуанг Т.С. и др. Быстрые алгоритмы в цифровой обработке изображений. - М.: Радио и связь, 1984. - 224 с.

[25] Сойфер В.А. Компьютерная обработка изображений. Часть 2. Методы и алгоритмы. - Соросовский образовательный журнал №3, 1996.

[26] Апальков И.В., Хрящев В.В. Удаление шума из изображений на основе нелинейных алгоритмов с использованием ранговой статистики. - Ярославский государственный университет, 2007.

[27] Радченко Ю.С. Эффективность приема сигналов на фоне комбинированной помехи с дополнительной обработкой в медианном фильтре. - "Журнал радиоэлектроники", №7, 2001. / http://jre.cplire.ru/iso/jul01/2/text.html

[28] Шликов В.В. Метод цифрової обробки відеоданих термограм при операціях на відкритому серці з фільтрацією візуальних фонів міокарда // Наукові вісті НТУУ "КПІ", о 1 (2018). $\quad$ C $26 \quad-\quad 36 . \quad-30$ URL: http://bulletin.kpi.ua/article//download/118807/pdf_286

[29] Wang C, Li T. An improved adaptive median filter for Image denoising. ICCEE 2012; 53(2.64): 393-8.

[30] Шлыков В.В., Данилова В.А., Диденко М.С. Пирометрическая система контроля температуры. Вісник Нац. техн. ун-ту "ХПІ": зб. наук. пр. Серія: Механікотехнологічні системи та комплекси. 2017. № 20 (1242). С. 11 $-19$.

[31] Лукин С.Б. Оптико-электронные системы: Конспект лекций. ИТМО, 2004. - СПб, ИТМО ИФФ, 2004. http://iff.ifmo.ru/kons/oes/KL.htm

[32] Лукин А. Введение в цифровую обработку сигналов (Математические основы).- М.: МГУ, Лаборатория компьютерной графики и мультимедиа, 2002. - URL: http://pv.bstu.ru/dsp/dspcourse.pdf

[33] Иванов Д.В. и др. Алгоритмические основы растровой графики. - Интернет университет информационных технологий. - http://www.intuit.ru/goto/course/ rastrgraph 


\section{УСУНЕННЯ ІМПУЛЬСНИХ ПЕРЕШКОД І ФЛУКТУАЦЙНИХ ШУМІВ В СИГНАЛІ ПІРОМЕТРА НА ОСНОВІ МЕТОДУ ДВОВИМІРНОГО СКАНУВАННЯ}

Шликов В.В., доц., к.Т.н. v.shlykov@kpi.ua

м. Київ, Україна

Данілова В.А., ст. викладач valnaa@ukr.net

Кафедра біомедичної інженерії Національний технічний університет України «Київський політехнічний інститут імені Ігоря Сікорського»

Реферат -Запропоновано метод попередньої обробки ицирових даних, отриманих при реєстрачії температури біологічного об'єкта пірометром, який дозволяє усунути в корисному сигналі флуктуаційні шуми і імпульсні перешкоди, обумовлені різною регулярною структурою геметріі фотоелектронних елементів оптичної матриці або структурою каналу передачі сигналу.

Ключові слова - температурний контроль, флуктуачійний шум, імпульсна перешкода, подавлення шумів, пірометр, кардіохірургія, біологічний об'єкт.

UDC 616.1-616.7

\section{ELIMINATION OF PULSE INTERFERENCE AND FLUCTUATION NOISES IN THE PYROMETER SIGNAL BASED ON THE TWO-SCANNING METHOD}

Shlykov V.V., Associate Professor, Ph.D. v.shlykov@kpi.ua

Kiev, Ukraine

Danilova V.A., Senior Lecturer. valnaa@ukr.net

Department of Biomedical Engineering National Technical University of Ukraine "Igor Sikorsky Kyiv Polytechnic Institute"

\footnotetext{
Abstract - A method is proposed for preliminary processing of digital data obtained by recording the temperature of a biological object with a pyrometer, which allows to eliminate fluctuation noises and impulse noise in a useful signal due to different regular hemetry structures of photoelectronic elements of the optical matrix or the structure of a signal transmission channel.
} 